\title{
PARAMETRIC ANALYSES OF NATURAL FREQUENCIES OF LOWER VIBRATION MODES OF SUSPENSION BRIDGES
}

\author{
By Masahiro YONEDA* and Manabu ITO**
}

\begin{abstract}
It is well known that lower modes of vibration are predominant in the dynamic response of suspension bridges to moving vehicles or wind. Therefore, it is needed to know their characterisics, in particular the corresponding natural frequencies.

In this brief paper, natural frequencies of lower vibration modes of suspension bridges are numerically examined, mainly with respect to a side to center span ratio besides nondimensional characteristic parameters introduced in the previous paper 2).

It is found from these results that the characteristics of symmetric natural frequencies of suspension bridges associated with lower modes depend significantly on the values of the side to center span ratio.

Keywords: suspension bridge, natural frequency, aerodynamic stability
\end{abstract}

\section{INTRODUCTION}

Lower modes of vibration are predominant in the dynamic response of a suspension bridge to moving vehicles or wind. The second author of this paper ${ }^{1)}$ once investigated the characteristics of the corresponding natural frequencies of lower vertical modes by introducing some nondimensional characteristic parameters and applied the results to the dynamic response analysis to the moving vehicles. Recently, the authors ${ }^{2)}$ conducted a series of parametric analysis for the natural frequencies of lower vertical and torsional modes of the suspension bridges with a side to center span ratio of 0.3 in order to investigate the effects of dead weight on the aerodynamic stability.

The characteristics of the lower mode shapes and the corresponding natural frequencies of a suspension bridge may be dependent on the side to center span ratio. Although Komatsu and Nishimura ${ }^{3)}$ discussed this span ratio aiming at finding the effects of shear deformation on higher modes of vibration, it seems that parametric studies observing the side to center span ratio have not been sufficiently available from aerodynamic viewpoint.

Hence, in this note, mainly with respect to a side to center span ratio besides nondimensional characteristic parameters introduced in reference 2), the natural frequencies of lower vibration modes of suspension bridges are parametrically analyzed in order to make up the previous paper 2) and discussed from aerodynamic viewpoint.

\section{NONDIMENSIONAL CHARACTERISTIC PARAMETERS}

The analytical method used here is based on the linerized deflection theory where horizontal cable

* Member of JSCE, M. Eng., Kawada Industries Inc. (Kita-ku, Tokyo)

** Member of JSCE, Dr. Eng., Prof. of Civil Eng., Univ. of Tokyo (Bunkyo-ku, Tokyo) 
tension is assumed constant, and the flexibility of towers as well as the influence of hanger inclination are neglected. This treatment may be justified in preliminary calculation.

The nondimensionalized circular frequencies of asymmetric vertical and torsional modes in the center span of a suspension bridge can be expressed by Eqs. (1) and (2), respectively.

For Asymmetric vertical modes : $\omega_{n, \eta} \sqrt{\frac{w l^{4}}{g E I}}=(n \pi)^{2} \sqrt{1+\left\{l \sqrt{\frac{H_{w}}{E I}}\right\}^{2} \frac{1}{(n \pi)^{2}}}$.

For Asymmetric torsional modes : $\omega_{n, \varphi} \sqrt{\frac{I_{\theta} l^{2}}{G J}}=(n \pi)^{2} \sqrt{\frac{1}{(n \pi)^{2}}+\left\{\frac{H_{w}\left(\frac{b}{2}\right)^{2}}{G J}\right\} \frac{1}{(n \pi)^{2}}}$

$$
(n=2,4 \cdots \cdots)
$$

where $\omega_{n, \eta}=$ natural circular frequency of vertical mode ; $\omega_{n, \varphi}=$ natural circular frequency of torsional modes ; $w=$ dead weight of the bridge per unit length ; $l=$ length of center span ; $H_{w}=$ horizontal component of the cable tension; $E I=$ flexural rigidity of the stiffening frame ; $I_{\theta}=$ polar moment of inertia of the suspension bridge per unit length; $G J=$ torsional rigidity of the stiffening frame ; $b=$ distance of the cables ; $g=$ gravity acceleration.

From the above formula, it is observed that the asymmetric modes of vertical and torsional vibration are respectively dependent on the parameter $P_{1, v}=l \sqrt{\frac{H_{w}}{E I}}$ and $P_{1, r}=\frac{H_{w}}{G J}\left(\frac{b}{2}\right)^{2}$ introduced in reference 2).

On the other hand, the nondimensional characteristic parameter $P_{2}=\frac{E_{c} A_{c}}{L_{E}} \frac{f^{2}}{H_{w} l}, P_{3}=\frac{l_{1}}{l}, P_{4}=\frac{w_{1}}{w}$ and $P_{5}=\frac{I_{1}}{I}$ defined in reference 2) appear in the frequency equations for symmetric modes. Furthermore in this paper, the following two nondimensional characteristic parameters concerned with the torsional vibrations of the suspension bridge with side spans shall be newly introduced in addition to the above-mentioned parameters.

$$
P_{6}=\frac{J_{1}}{J}, \quad P_{7}=\frac{I_{\theta 1}}{I_{\theta}}
$$

where $J=$ torsional constant of the center span. Index " 1 " represents the quantity at side spans. $P_{6}$ and $P_{7}$ were taken to be 1.0 in the previous paper.

\section{NUMERICAL ANALYSIS AND DISCUSSION}

In what follows the numerical analysis and discussions will be made on the first symmetric and asymmetric mode of natural vibrations, mainly from aerodynamic viewpoint.

\section{(1) Vertical vibration}

Fig. 1 shows the nondimensionalized natural circular frequencies of the single span, two hinged suspension bridges $\left(P_{3}=0\right)$. Figs. 2 and 3 show those of the three span, two hinged suspension bridges with a side to center span ratio $P_{3}$ of 0.35 and 0.50 , respectively, when $P_{4}$ and $P_{5}$ are taken to be 1.0 . From these figures, it can be seen that for $P_{1}, v$ larger than 10, the first symmetric mode gives the lowest natural frequency in case of $P_{3}$ larger than about 0.35 , whereas the first asymmetric mode gives the lowest natural frequency in case of $P_{3}$ less than about 0.35 . Furthermore, it is noticed that according to the increase of $P_{3}$, the effects of $P_{2}$ on the first symmetric vibration are decreased. For simplicity, let us consider the case that the dead weight $w$ per unit length of a suspension bridge and flexural rigidity EI change independently. The horizontal component $H_{w}$ of cable tension is proportional to the dead weight $w . P_{2}$ does not change much when the dead weight increases, because the sectional area Ac of cable increases almost in proportion to the magnitude of $H_{w}$. The ratio $\Delta \omega_{2}^{*} / \Delta P_{1}, v$ of the respective change rates of $\omega_{2}^{*}$ (nondimensionalized natural circular frequency of vertical mode) and $P_{1}, v$ is, within the range of $P_{3}$ less that 0.5 , only somewhat smaller than 1.0 for both the symmetric and asymmetric vertical mode when $\Delta P_{1}, v$ is equal to 2.0. Therefore, it is surmized that the natural frequencies of the first symmetric 


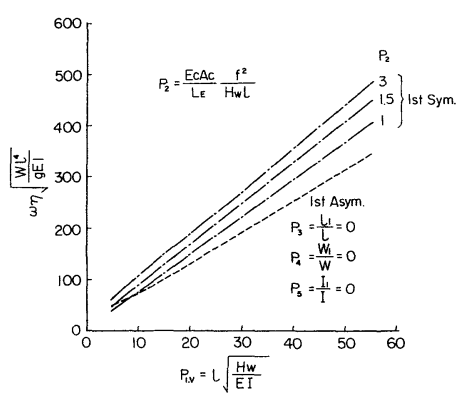

Fig. 1 Nondimensionalized Vertical Frequencies $\left(P_{3}=\frac{l_{1}}{l}=0\right)$.

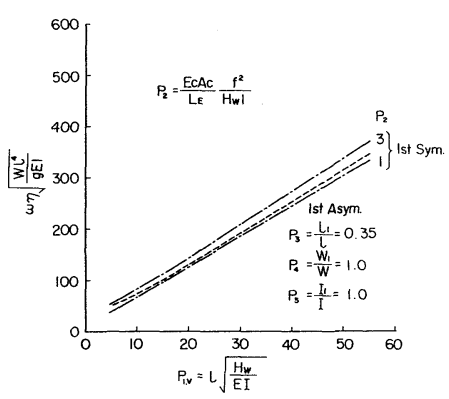

Fig. 2 Nondimensionalized Vertical Frequencies $\left(P_{3}=\frac{l_{1}}{l}=0.35\right)$.

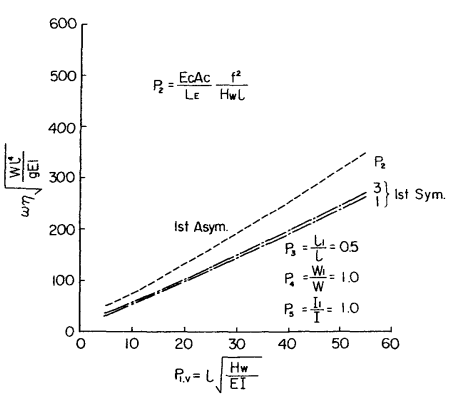

Fig. 3 Nondimensionalized Vertical Frequencies $\left(P_{3}=\frac{l_{1}}{l}=0.5\right)$.

and asymmetric vertical mode scarcely affected by $P_{1}, v$, when $P_{4}$ and $P_{5}$ are assumed to be equal to 1. 0 . On the other hand, $H_{w}$ also increases in inverse proportion to the center span sag $\mathrm{f}$. Therefore, when the center span sag $f$ is lessened in order to increase $H_{w}, P_{2}$ becomes small, as can be seen from its definition, but the frequencies of the first symmetric and asymmetric vertical mode change only slightly because the dead weight of cable does not remarkably increase.

The effects of both $P_{4}$ and $P_{5}$ on the frequencies of symmetric modes of suspension bridges will be considered next. From the reuslts of calculation, it is found that nondimensionalized natural circular frequencies of the symmetric modes are a little reduced with the increase of $P_{4}$, and little changed with the increase of $P_{5}$.

\section{( 2 ) Torsional vibration}

Fig. 4 shows the nondimensionalized natural circular frequencies of the single span, two hinged suspension bridges $\left(P_{3}=0\right)$. Figs. 5 and 6 show those of the three span, two hinged suspension bridges with a side to center span ratio of 0.35 and 0.5 , respectively, when $P_{4}, P_{6}$ and $P_{7}$ are taken to be 1.0 . From these figures, it can be seen that the first symmetric mode gives the lowest natural frequency when $P_{3}$ is more than about 0.35 . It is also observed that when $P_{3}$ is less than about 0.35 , it depends on the value of $P_{1, T}$ and $P_{2}$ whether first symmetric or asymmetric mode gives the lowest natural frequency. When both $P_{1, T}$ and $P_{2}$ are small, the symmetric mode is the fundamental mode of vibration, and vice versa. Moreover, the nondimensionalized natural circular frequency of the first asymmetric mode increases almost lineraly to the increase of $P_{1, r}$. On the other hand, although the same findings can be applied for the nondimensionalized natural circular frequency of first symmetric mode, the degree of increase in the frequency is larger for the smaller values of $P_{1, T}$. The effects of $P_{2}$ on nondimensionalized natural circular frequency of the first symmetric mode decreases with the increase of $P_{3}$. As mentioned before, the

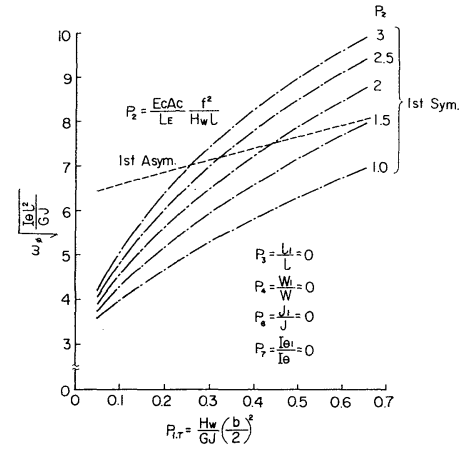

Fig. 4 Nondimensionalized Torsional Frequencies $\left(P_{3}=\frac{l_{1}}{l}=0\right)$.

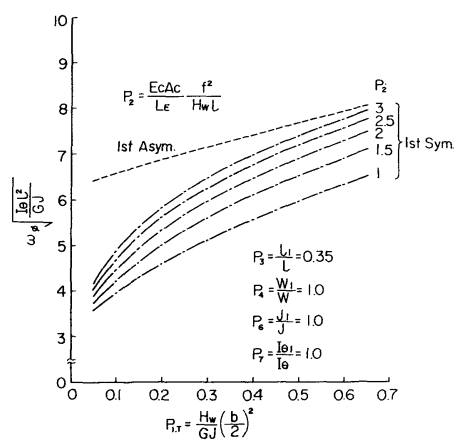

Fig. 5 Nondimensionalized Torsional Frequencies $\left(P_{3}=\frac{l_{1}}{l}=0.35\right)$.

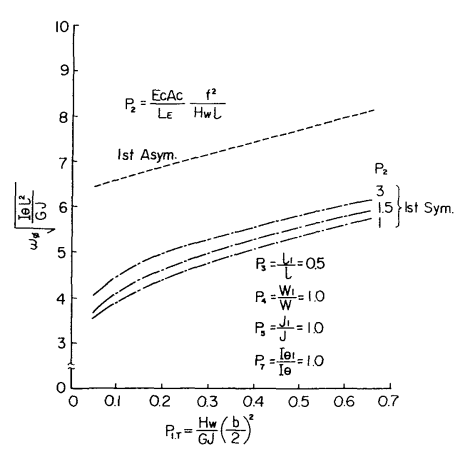

Fig. 6 Nondimensionalized Torsional Frequencies $\left(P_{3}=\frac{l_{1}}{l}=0.5\right)$. 
horizontal cable tension $H_{w}$ increases in proportion to the dead weight $w$ and the sectional area $A_{c}$ of the cable increases almost linerly in proportion to the magnitude of $H_{w}$. Accordingly, the polar moment of inertia $I_{\theta}$ also increases. If the torsional rigidity $G J$ is augmented, the dead weight and polar moment of inertia increase to some extent. But, only the effects of the torsional rigidity $G J$ is considered here for simplicity. In this connection, calculation of $\Delta \omega_{\varphi}^{*} / \sqrt{\Delta P_{1, T}}$, where $\Delta \omega_{\varphi}^{*}$ and $\Delta P_{1, \tau}$ are the change rates of $\omega_{\varphi}^{*}$ (nondimensionalized natural circular frequency of torsional mode) and $P_{1, T}$, respectively, was made in order to grasp the characteristics of the torsional vibration when $P_{3}$ is 0.35 and $P_{1, T}$ is within the range of less than about 0.35 , it was found that this value was somewhat smaller than 1.0 when $\Delta P_{1, T}$ is equal to 2.0. Furthermore, this value is nearly equal to 1.0 when $P_{3}$ is 0 and $P_{1, T}$ is within the same range. Therefore, it is surmized that the natural frequency of first symmetric torsional mode in the range of both $P_{3}$ less than about 0.35 and $P_{1, T}$ less than about 0.35 does not drastically affect by parameter $P_{1, T}$ when $P_{4}$, $P_{6}$ and $P_{7}$ are assumed to be equal to 1.0 . When the center span sag $f$ is lessened in order to increase $H_{w}$, $P_{2}$ becomes small, as can be seen from its definition, so that, the frequencies of the first symmetric mode is expected to be reduced sensitively in the small value of $P_{3}$.

On the other hand, in case of the asymmetric torsional frequency, the ratio $\Delta \omega_{\varphi}^{*} / \sqrt{\Delta P_{1, T}}$ was about 0.75 when $\Delta P_{1, T}$ is equal to 2.0 . Therefore, it can be said that the asymmetric torsional frequency sensitively affects by parameter $P_{1, r}$. The same findings can be applied for the symmetric torsional frequencies in the range of about both $P_{3}$ larger than about 0.35 and $P_{1, T}$ larger than about 0.35 .

Finally dealt with are effects of the parameters $P_{4}, P_{6}$ and $P_{7}$ on symmetric vibration modes of suspension bridges. From the results of calculation, it is found that nondimensionalized natural circular frequencies of symmetric modes are a little reduced with the increase of $P_{4}, P_{7}$ and a little increases with the increase of $P_{6}$.

\section{CONCLUDING REMARKS}

Main conclusions are summerized as follows.

(1) The first symmetric mode of vertical vibration gives the lowest natural frequency when $P_{3}$ is larger than about 0.35 . On the other hand, the first asymmetric mode of vertical vibration gives the lowest natural frequency when $P_{3}$ is less than about 0.35 . Furthermore, both the frequencies of first symmetric and asymmetric mode little affect by parameter $P_{1, v}$.

(2) The first symmetric mode of torsional vibration gives the lowest natural frequency when $P_{3}$ is larger than about 0.35 . On the other hand, it depends on the values of $P_{1, T}$ and $P_{2}$ whether first symmetric or asymmetric mode gives the lowest natural frequency when $P_{3}$ is less than about 0.35 . Furthermore, there are ranges of the nondimensional characteristic parameters in which the first symmetric frequency does not drastically affect by parameter $P_{1, T}$.

(3) The effects of $P_{2}$ on both the vertical and torsional frequencies of the first symmetric mode increase with the decrease of $P_{3}$. Therefore, it is noteworthy from aerodynamic viewpoint that the small side to center span ratio and the large value of center span sag can raise the first symmetric torsional frequency.

Finally, the authors would like to thank Dr. Ken-ich Maeda, Kawada Industries Inc., for his valuable advice.

\section{REFERENCES}

1) Ito, M. : Response of Suspension Bridges to Moving Vehicles, Trans. of JSCE, Vol. 149, Jan., 1968. (in Japanese).

2) Yoneda, M. and Ito, M. : Effects of Dead Weight on Aerodynamic Stability of Long-Span Suspension Bridges, Proc. of JSCE, No. 368/I-5, Apr., 1986.

3) Komatsu, S. and Nishimura, N. : Effects of Shear Deformation on Free Vibration of Long Span Suspension Bridges, Proc. of JSCE, No. 323, Jul., 1982 (In Japanese). 